\title{
Transmembrane NADH oxidation with tetracyanoquinodimethane
}

\author{
MinHui Wang ${ }^{a \dagger}$, Christian Wölfer ${ }^{b \dagger}$, Lado Otrin ${ }^{a}$, Ivan Ivanov ${ }^{a^{*}}$, Tanja Vidaković- \\ Koch $^{\text {a }}$, Kai Sundmacher ${ }^{\mathrm{a}, \mathrm{c}}$ \\ ${ }^{a}$ Max Planck Institute for Dynamics of Complex Technical Systems, Process Systems Engineering, Sandtorstr.1, \\ 39106 Magdeburg, Germany \\ ${ }^{b}$ Max Planck Institute for Dynamics of Complex Technical Systems, Process Synthesis and Process Dynamics, \\ Sandtorstr. 1, 39106 Magdeburg, Germany \\ ${ }^{c}$ Otto-von-Guericke University Magdeburg, Department of Process Systems Engineering, Universitätsplatz 2, \\ 39106 Magdeburg, Germany \\ * corresponding author ivanov@mpi-magdeburg.mpg.de \\ ${ }^{\dagger}$ both authors contributed equally to this work
}

\begin{abstract}
The design of efficient schemes for nicotinamide adenine dinucleotide (NAD) regeneration is essential for the development of enzymatic biotechnological processes in order to sustain continuous production. In line with our motivation for encapsulation of redox cascades in liposomes to serve as microbioreactors, we developed a straightforward strategy for interfacial oxidation of entrapped NADH by ferricyanide as external electron acceptor. Instead of the commonly applied enzymatic regeneration methods, we employed hydrophobic redox shuttle embedded in the liposome bilayer. Tetracyanoquinodimethane (TCNQ) mediated electron transfer across the membrane and thus allowed us to shortcut and to emulate part of the electron transfer chain functionality without the involvement of membrane proteins. To describe the experimental system we developed a mathematical model, which allowed for determination of rate constants and exhibited handy predictive utility.
\end{abstract}

Keywords: electron transfer, mediator, phospholipid membrane, NAD regeneration, TCNQ.

\section{Introduction}

Due to its prime function as a cofactor in redox reactions, nicotinamide adenine dinucleotide (NAD) is a vital regulator in living cells and is thus related to fundamental processes such as transcription, cell cycle and apoptosis ${ }^{1,2,3}$. Regarding its immediate role in metabolism, the significance of NAD arises from its abundance - most of the known oxidoreductases depend on $\mathrm{NAD}(\mathrm{P})$. The required stoichiometric quantities render many, otherwise elegant, 
biotransformation pathways uneconomic. Therefore, the expanding field of enzymatic biotechnology is seeking for efficient ways for NAD regeneration ${ }^{4,5}$. Along with this biotechnological perspective, our rationale comes from a bottom-up synthetic biology standpoint. The reductionist view of the cell ${ }^{6}$ as a set of metabolic reactions in a confined space has designated the encapsulation of enzymes in phospholipid vesicles as one of the main workhorses in this research field ${ }^{7,8}$. The assembly of such liposome bioreactors is still in its infancy due to the intrinsic complexity of metabolic networks and the difficulties in realizing transport mechanisms for reactants and products. Another major hurdle is the supply of cofactors such as $\mathrm{NAD}(\mathrm{P})$ and $\mathrm{ATP}$, which virtually represent the energy needed to drive the reactions. To the best of our knowledge, no schemes for direct cofactor transport through the lipid membrane (e.g. through reconstitution of specific cofactor transporters) have been devised until now. Thus, studies in the framework of artificial cells have been generally limited to easy-to-follow model reactions, DNA-related reactions and whole-cell extracts ${ }^{8,9,10}$. The entrapment of dehydrogenases for more practical applications such as stabilization has been largely overlooked ${ }^{11}$ and the few existing reports relied on the limited quantity of initially entrapped $\mathrm{NAD}^{12,13,14}$. On the contrary, strategies for continuous $\mathrm{NAD}^{+}$regeneration in liposomes have been employed for energy conversion systems, similar to the concept of biofuel cells. Kano and coworkers constructed "artificial microbes" through entrapment of dehydrogenases and a diaphorase as biocatalyst for NADH oxidation, and demonstrated the generation of electrical current with ethanol ${ }^{15}$ and glucose ${ }^{16}$. Regeneration of diaphorase was accomplished through electrochemical oxidation of 2,3-dimethoxy-5-methyl-1,4benzoquinone (coenzyme $\mathrm{Q}_{0}$ ) as diffusional mediator, which freely penetrated the liposomes.

With the ultimate goal of reconstituting practically relevant metabolic cascades in vesicles, we sought for ways to oxidize entrapped NADH by redox agents placed outside of the compartment, in order to maintain a minimal composition and to avoid undesired interactions (e.g. with the components of enzymatic regeneration systems ${ }^{4}$ ). Therefore, we intentionally refrained from architectures, employing enzymes and mediators (oxidants) present in the liposome interior, which were previously applied ${ }^{15}$, to create a minimal and versatile regeneration chassis without the involvement of additional proteins. Thus, we aimed for a transmembrane oxidation scheme, which would keep the oxidant to the vesicle exterior and would resemble the natural setup of electron transport chains. Studies on transmembrane electron transfer have historically strived for elucidation of the physical principles behind electron transfer chains (oxidative phosphorylation and photosynthetic) and have resulted in numerous examples of reduced in vitro models ${ }^{17}$. In a founding experiment to construct a 
simple model system for the chemiosmotic hypothesis Hinkle investigated the mediated reduction of ferricyanide by ascorbate and thereby implicitly postulated certain requirements for transmembrane electron transfer mediators ${ }^{18}$. The majority of similar works was motivated by fundamental interest in electron transfer with an emphasis on photo-transducers for energy conversion, and in very few cases straightforward implementation was proposed. As far as we know, the only example of a conceptually akin, application-driven assembly was the reduction of encapsulated methemoglobin by NADH in the context of hemoglobin vesicles as red-cell substitutes $^{19}$.

In a similar fashion, we developed a method for oxidation of entrapped NADH through a mediator embedded in the membrane. Thus, we reproduced and streamlined through a distinct form of interfacial redox catalysis the simultaneous pyridine nucleotide oxidation and transmembrane electron transfer, functionalities typically exclusive for biological systems (Fig. 1).

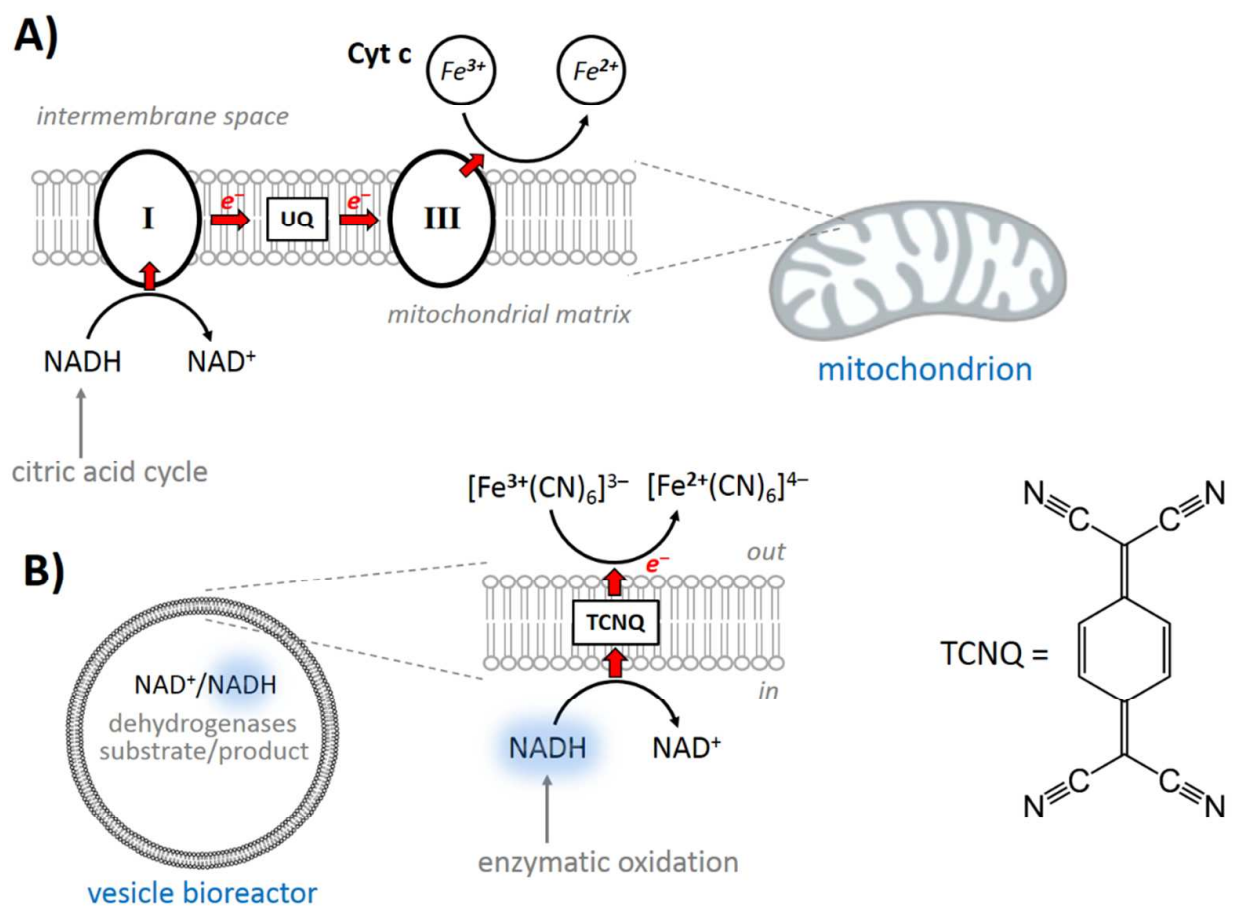

Figure 1: Schematic presentation of the analogy between transmembrane electron transfer in biological systems and in the present case. Electron flow shown in red block arrows. A) in the mitochondrial electron transport chain NADH-ubiquinone oxidoreductase (Complex I) oxidizes NADH (product from metabolism, e.g. citric acid cycle) and reduces ubiquinone (UQ) in the bilayer; the resulting ubiquinol is then re-oxidized by quinol-cytochrome-c oxidoreductase (Complex III), which also reduces Fe(III) in the heme group of cytochrome c (Cyt c). B) NADH encapsulated in liposome (product of prospective enzymatic oxidation) is oxidized by mediator such as TCNQ embedded in the bilayer; reduced TCNQ is then re-oxidized by Fe(III) in the ferricyanide complex. In both cases electrons are transferred from the interior to the exterior but in the present case this process occurs without the involvement of Complexes I and III. 


\section{Materials and methods}

\section{Materials}

1-palmitoyl-2-oleoyl-sn-glycero-3-phosphatidylcholine (POPC) from Avanti Polar Lipids Inc. (Alabaster, AL, USA) was dissolved in chloroform to a concentration of $100 \mathrm{mg} \mathrm{mL}^{-1}$ and used as a stock solution. $\mathrm{NAD}^{+}$and NADH-disodium salt were purchased from Carl Roth $\mathrm{GmbH}$ (Karlsruhe, Germany). Potassium ferricyanide $\left(\mathrm{K}_{3}\left[\mathrm{Fe}(\mathrm{CN})_{6}\right]\right)$ was purchased from Merck KGaA (Darmstadt, Germany). All other chemicals including 7,7,8,8tetracyanoquinodimethane (TCNQ), ubiquinone 10, Nile Red, 5(6)-carboxyfluorescein and Triton X-100 10\% solution were purchased from Sigma-Aldrich Chemie GmbH (Steinheim, Germany). Octyl $\beta$-D-glucopyranoside was purchased from GLYCON Biochemicals GmbH (Luckenwalde, Germany). LiTCNQ was synthesized according to the literature ${ }^{20}$. Deionized water was obtained from a Milli-Q water purification system (Millipore Corp., Billerica, MA, USA).

\section{Preparation of vesicles}

Three different hydrophobic mediators were reconstituted in the bilayer membrane and $\mathrm{NADH}$ or carboxyfluorescein were encapsulated in the hydrophilic interior of vesicles, prepared by extrusion. In the general procedure $40 \mu \mathrm{L}$ of POPC stock solution were diluted with $200 \mu \mathrm{L}$ chloroform for better dispersion and the mixture was spread on the bottom of a glass tube by a pipette. For preparation of vesicles with mediator $80 \mu \mathrm{L}\left(1 \mathrm{mg} \mathrm{mL} \mathrm{m}^{-1}\right.$ ubiquinone in chloroform), $66 \mu \mathrm{L}\left(0.3 \mathrm{mg} \mathrm{mL}^{-1} \mathrm{TCNQ}\right.$ in acetonitrile $)$ or $64 \mu \mathrm{L}\left(0.5 \mathrm{mg} \mathrm{mL}^{-1}\right.$ Nile Red in methanol) were added to the POPC solution before drying, maintaining a 50:1 molar ratio between lipids and mediator in order to preserve bilayer integrity. Then the lipid layer was dried under gentle nitrogen flow and vacuum for $1 \mathrm{~h}$. Afterwards the resulting thin films were rehydrated by adding $500 \mu \mathrm{L}$ MOPS buffer (100 mM MOPS, $250 \mathrm{mM} \mathrm{KCl}$, Tris, $\mathrm{pH}$ 7.2), which contained $10 \mathrm{mM} \mathrm{NADH}$ or $10 \mathrm{mM}$ carboxyfluorescein when necessary. To detach the film from the tube, the hydration solution was pipetted against the tube wall multiple times and subjected to short vortex cycles in order to resuspend homogeneously. Then the suspensions were treated in repetitive freeze-thaw cycles ( $>6$ times), whereby each cycle consisted of freezing in liquid nitrogen for 1-2 min and thawing for 2-4 min in $40{ }^{\circ} \mathrm{C}$ water bath. In some cases an aliquot of the resulting suspensions was imaged on Axio imager A1 microscope (Carl Zeiss, Germany) in order to follow the formation of vesicles and the presence of mediator in the membrane. In order to obtain large unilamellar vesicles $(<200 \mathrm{~nm})$ 
of uniform size, the lipid suspensions were filtered $>11$ times by Avanti extruder (Avanti Polar Lipids, Inc., Alabaster, AL, USA) through Nuclepore Track-Etched Membrane filter $(0.2 \mu \mathrm{m}$ pore size, Whatman PLC, UK). The filtrate, containing vesicles, was collected and stored at $4{ }^{\circ} \mathrm{C}$ for later use.

When NADH or carboxyfluorescein were encapsulated, the free compounds in the hydration solution were separated by column chromatography through a disposable PD-10 desalting column (GE Healthcare Life Sciences, Chicago, IL, USA) filled with $8.3 \mathrm{~mL}$ Sephadex G-25 matrix $(\varnothing 14.5 \mathrm{~mm} \mathrm{ca} .50 \mathrm{~mm})$. The filtrate containing purified vesicles was collected and stored at $4{ }^{\circ} \mathrm{C}$.

The size of vesicles was determined by dynamic light scattering (DLS) using a Zetasizer Nano ZS (Malvern, Worcestershire, UK) with a $663 \mathrm{~nm} \mathrm{He}-\mathrm{Ne}$ laser at a fixed scattering angle of $173^{\circ}$ at $25^{\circ} \mathrm{C}$. Every measurement was run three times with $70 \mathrm{~s}$ duration of each single run and the average was obtained. In addition the size and concentration of vesicles were determined by tunable resistive pulse sensing (TRPS) on a qNano instrument (Izon Science Ltd., Christchurch, New Zealand). For the measurement a NP200 stretchable nanopore was used, which was calibrated with carboxylated polystyrene beads (CPC400F, mean size $350 \mathrm{~nm}$, concentration $6 \times 10^{11}$ particles $\mathrm{mL}^{-1}$ ). The lower fluid cell was filled with $75 \mu \mathrm{l}$ MOPS buffer and $35 \mu \mathrm{l}$ of the vesicle suspension in MOPS buffer were added to the upper cell. Size distribution and concentration were calculated from the measurement data with the instrument software (Izon Control Suite 2, Chrsitchurch, New Zealand).

\section{Transmembrane electron transfer experiments}

The oxidation of NADH was evaluated by monitoring the fluorescence intensity of NADH on Cary Eclipse Fluorescence Spectrophotometer (Agilent Technologies, Santa Clara, CA, USA). Briefly, vesicles with different mediators, reconstituted in the membrane and encapsulated NADH were placed in a $10 \mathrm{~mm}$ quartz cuvette $(500 \mu \mathrm{L}$, Hellma, Germany) at room temperature and the fluorescence was monitored for over $12 \mathrm{~h}$ at $460 \mathrm{~nm}$ (slit width: 20) at excitation wavelength of $340 \mathrm{~nm}$ (slit width: 20) after the addition of different amounts of ferricyanide $(100,200$, and $400 \mu \mathrm{M})$. Vesicles lacking mediators and/or NADH were also monitored under the same conditions as controls. In the case of leakage experiments the carboxyfluorescein fluorescence was monitored at $524 \mathrm{~nm}$ (slit width: 2.5) at excitation wavelength of $494 \mathrm{~nm}$ (slit width: 2.5). 


\section{Results and discussion}

\section{Screening of transmembrane electron transfer mediators}

Despite the substantial difference between the redox potentials of NADH and oxygen $(0.82$ $\mathrm{V}^{21}-\left(-0.32 \mathrm{~V}^{22}\right)=1.14 \mathrm{~V}$ at standard conditions, $25{ }^{\circ} \mathrm{C}$ and $\left.\mathrm{pH} 7\right), \mathrm{NADH}$ is stable against oxygen unless either species are activated. On the contrary, reaction of NADH with ferricyanide readily takes place when the two components are in the same aqueous phase (Fig. S1). Ferricyanide is a convenient and widely used oxidant with high water solubility, unlike oxygen. The ferri/ferrocyanide redox couple has a long history in transmembrane electron transfer studies ${ }^{17}$ and can be traced back to some fundamental experiments in this field ${ }^{18}$. In addition, it is easily regenerated electrochemically, thus providing possible means for the use of electrical energy to drive biological reactions as shown in the literature ${ }^{15,16,23}$.

In the present case, apart from ion carrier properties, favorable redox potential and lipophilic partitioning, we additionally aimed for favorable kinetics towards NADH. The first apparent candidate was ubiquinone 10 (coenzyme $\mathrm{Q}_{10}$, UQ), which acts as an electron transfer mediator within the hydrophobic region of the lipid membrane as part of the respiratory chain in mitochondria. UQ has been found to stimulate electron transfer in free-standing bilayers ${ }^{24}$ and to mediate NAD redox conversions in supported and tethered bilayers ${ }^{25,26}$. TCNQ is another mediator with a long history in electron transfer research, which has been extensively studied by electrochemical techniques in free-standing ${ }^{27,28}$ and supported bilayers ${ }^{29}$, and was also used in amperometric sensor for NADH determination ${ }^{30}$. Similarly to UQ, TCNQ partitions exclusively in the membrane, evidenced by the estimated partition coefficient of $520000^{31}$. In addition to the previous two molecules we hypothesized that Nile Red, a common lipid stain, could also be employed because of the structural similarity to other phenoxazine and phenothiazine dyes with known activity for NADH oxidation ${ }^{32,33}$.

In order to test the validity and effectiveness of our approach for transmembrane NADH oxidation, we produced large unilamellar vesicles, containing NADH in the interior, whereas the respective mediators were embedded in the bilayer. To prove localization of the mediators in the membrane, after rehydration the lipid suspensions were imaged on a fluorescence microscope. While reduced UQ exhibits intrinsic fluorescence in liposomes with a peak around $370 \mathrm{~nm}^{34}$, it was not possible to observe the oxidized form under a microscope. On the contrary, TCNQ is fluorescent in non-polar solvents and the intensity decreases fast with increasing solvent polarity ${ }^{35}$. Due to the broad emission spectrum it was possible to observe both green and blue fluorescence of the vesicle suspension before extrusion, which indicated 
successful localization of TCNQ in the hydrophobic region of the bilayer (Fig. S2). Nile Red on the other side exhibited bright red/orange fluorescence as expected (data not shown).

Based on DLS measurements, the average diameters of different vesicle preparations matched well the filter pore size of the extruder and the low polydispersity index in the range 0.1-0.2 indicated monodisperse distributions. In addition, mean diameter and concentration of vesicles were determined by TRPS (Table S1). NADH encapsulation and subsequent column purification did not change vesicle size, compared to vesicles containing only mediator. The reconstitution of UQ resulted in reduction of size to $c a .150 \mathrm{~nm}$ ( vs. $170 \mathrm{~nm}$ for the other mediators), which can be attributed to the increased density and order of the bilayer ${ }^{36}$.

All three mediators apparently stimulated the decrease of NADH fluorescence when 400 $\mu \mathrm{M}$ ferricyanide was added to the outer solution (Fig. 2). Since the amount of encapsulated NADH ( $\sim 15 \mathrm{nmol}$ in the sample, $1.6-5.2 \times 10^{-20} \mathrm{~mol} \mathrm{NADH}$ per vesicle) and the concentration of vesicles in the cuvette slightly varied ( $\pm c a .10 \%$ difference in the intensity) the curves were normalized to their initial values for more clear comparison (original data in Fig. S3). $\mathrm{NADH}$ is known to be unstable in certain aqueous solutions, whereby the most influential factors are $\mathrm{pH}$ and buffer type ${ }^{37}$. Therefore, we also monitored the stability of NADH, both free and encapsulated in mediator-free vesicles (Fig. 2) and found around 10\% decay in the course of the measurement. From the three mediators TCNQ exhibited the most prominent activity. In fact, the fluorescence decrease in the case of UQ and Nile Red cannot be unequivocally ascribed to the proposed mechanism due to the insufficient statistics of these preliminary screening experiments. Moreover, while TCNQ stimulated exponential decrease, the behavior in the other two cases was qualitatively similar to degradation, whereby a detrimental effect of the lipid phosphate groups can be anticipated ${ }^{37}$.

Regarding UQ, although Campos and Kataky deduced UQ-mediated electron transfer for the $\mathrm{NAD}^{+} / \mathrm{NADH}$ couple from cyclic voltammetry data in a black lipid membrane (BLM) setup, their quantitative analysis based on impedance spectroscopy resulted in a negligible change of the apparent electron transfer rate constant from $6.89 \pm 0.70 \times 10^{-8} \mathrm{~cm} \mathrm{~s}^{-1}$ in unmodified films to $7.81 \pm 0.78 \times 10^{-8} \mathrm{~cm} \mathrm{~s}^{-1}$ in UQ-modified ones ${ }^{25}$. Indeed, it would be puzzling if UQ in the mitochondrial membrane efficiently oxidized NADH since the proton pumping by Complex I would be bypassed in that way ${ }^{38}$. 


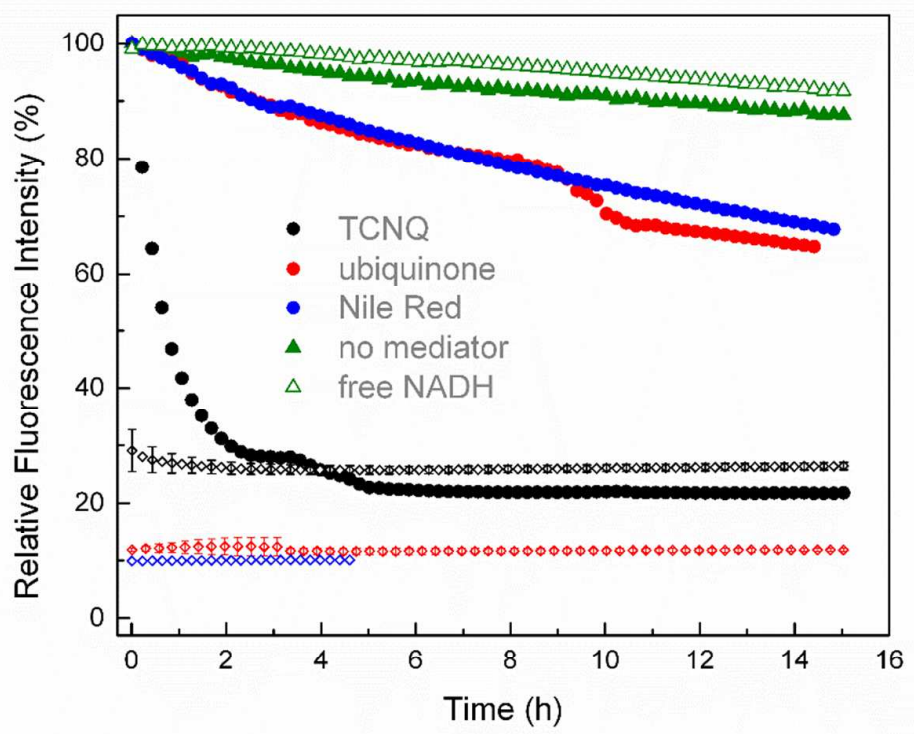

Figure 2: Relative fluorescence intensity profiles of different POPC vesicle suspensions at $460 \mathrm{~nm}$ : vesicles with reconstituted mediators + encapsulated $<10 \mathrm{mM} \mathrm{NADH}$ in presence of $400 \mu \mathrm{M}$ potassium ferricyanide (black, red and blue full dots); vesicles with reconstituted mediators only (black, red and blue empty diamonds); vesicles with encapsulated $<10 \mathrm{mM}$ NADH (green full triangles); free $20 \mu \mathrm{M} \mathrm{NADH}$ in solution (green empty triangles); $\sim 0.37 \mathrm{mM}$ POPC in $100 \mathrm{mM}$ MOPS, $250 \mathrm{mM} \mathrm{KCl}$, Tris, $\mathrm{pH} 7.2$.

In the case of TCNQ-mediated transmembrane electron transfer, the fluorescence of encapsulated NADH decreased to about $20 \%$ of the initial value after $5 \mathrm{~h}$ and did not change significantly afterwards. However, the residual fluorescence signal did not originate from unoxidized NADH but from a background signal. The latter was confirmed in control experiments with vesicles lacking NADH, normalized to the respective vesicle preparations containing NADH, as shown in Fig. 2. The small deviation in the case of TCNQ was due to the different vesicle concentrations in the samples. In addition, fluorescent spectra at the beginning and at the end of the experiment indicated complete disappearance of the characteristic NADH emission peak (Fig. S4). It should be noted that in order to increase the light output of the measurement to account for the low NADH concentration and weak intrinsic fluorescence, we used the maximum available slit widths, which respectively resulted in higher noise. Thus, empty UQ and Nile Red vesicles also exhibited about $10 \%$ of the full vesicles response (Fig. 2), while the background signal at $460 \mathrm{~nm}$ in the case of TCNQ vesicles was in addition augmented by the TCNQ fluorescence ${ }^{35}$.

Provided that NADH reacts with ferricyanide in bulk (Fig. S1), the decrease of NADH fluorescence might be ascribed to direct, unmediated oxidation of $\mathrm{NADH}$, released from the vesicles through membrane defects due to the presence of TCNQ. To rule out this possibility 
we performed control experiments with pristine and TCNQ-containing liposomes and assessed the respective leakage. Although dequenching of NADH has been previously used to determine liposome stability against ethanol ${ }^{15}$, we did not observe sufficient increase in fluorescence intensity after disrupting the vesicles with surfactants (about $7 \%$ difference at $460 \mathrm{~nm}$ ) to be able to quantify the possible leakage of NADH. In contrast, carboxyfluorescein (CF) exhibits sharp self-quenching (Fig. S5) and is therefore used in a standard method for assessment of liposome stability and permeability ${ }^{39}$. Based on the experimental limitations of direct NADH monitoring on the one side and the established use of the CF assay on the other side, alongside the smaller size of CF compared to NADH, we evaluated the influence of TCNQ on membrane permeability through CF as a model for encapsulated species.

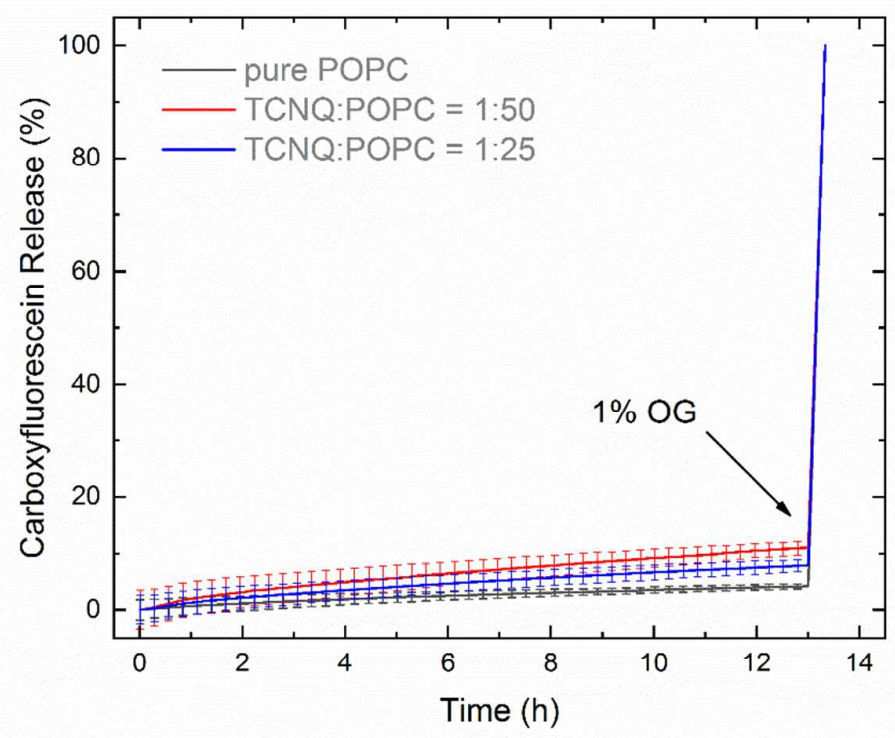

Figure 3: Fluorescence profiles of carboxyfluorescein encapsulated in POPC vesicles at $524 \mathrm{~nm}$, without or with embedded TCNQ; $\sim 0.37 \mathrm{mM}$ POPC in $100 \mathrm{mM}$ MOPS, $250 \mathrm{mM} \mathrm{KCl,} \mathrm{Tris,} \mathrm{pH} \mathrm{7.2.}$

In triplicate experiments the dye fluorescence was monitored for $13 \mathrm{~h}$ and afterwards $1 \%$ octyl glucoside (OG) was added in order to disrupt the vesicles and achieve complete release and dequenching. CF is known to spontaneously leak from POPC vesicles with a low rate but the precise mechanism is unknown ${ }^{40}$. The presence of TCNQ in the membrane resulted in a small increase of content release (Fig. 3), which could not account for the observed NADH oxidation rate (Fig. 2). In the relevant time frame of $5 \mathrm{~h}$, vesicles with 1:50 and 1:25 ratio of TCNQ to POPC exhibited $5.6 \pm 2.3 \%$ and $4.1 \pm 1.6 \%$ release, respectively, while pure POPC liposomes let out $2.2 \pm 0.9 \%$ of their content. 


\section{Modeling of TCNQ-mediated electron transfer}

Based on the mediator screening results we identified TCNQ as a promising catalyst for transmembrane NADH oxidation and we varied the concentration of ferricyanide to examine its effect. Decrease of ferricyanide resulted in slower NADH oxidation as shown in Fig. 4 (at least triplicate measurements).

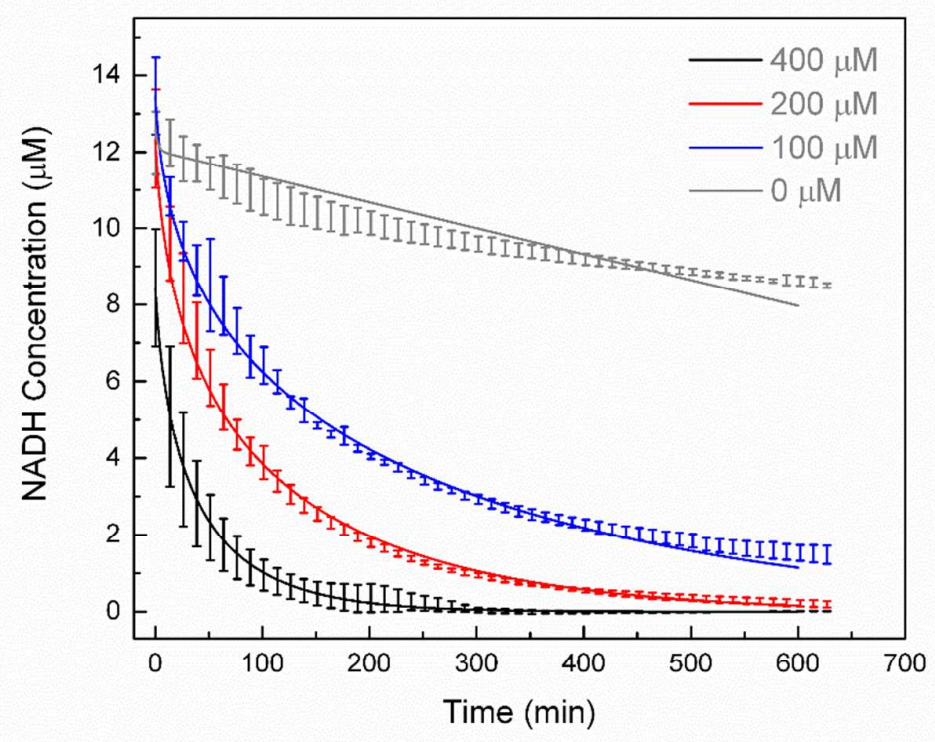

Figure 4: NADH concentration profiles (experimental average: error bars, and simulated: full lines) of POPC vesicle suspensions with reconstituted TCNQ in presence of different potassium ferricyanide concentrations; $\sim 0.37 \mathrm{mM}$ POPC in $100 \mathrm{mM}$ MOPS, $250 \mathrm{mM} \mathrm{KCl}$, Tris, $\mathrm{pH}$ 7.2.

In order to convert the fluorescence signal to concentration we used a calibration curve of free NADH (Fig. S6) and corrected for the background fluorescence of empty TCNQ vesicles. Despite the fact that the NADH calibration curve was not recorded at identical conditions (free $\mathrm{NADH}$ in the $\mu \mathrm{M}$ range versus encapsulated in the $\mathrm{mM}$ range) we assumed that its use was justified because we did not observe quenching effects in the relevant concentration range, neither in bulk experiments, nor after disrupting the vesicles with Triton ${ }^{\circledR}$. In addition, the total volume of vesicles estimated from TRPS data $\left(1.98 \times 10^{-3} \mathrm{ml}\right.$ in $\left.1 \mathrm{ml}\right)$ corresponded to the expected fluorescent signal when accounting for the inevitable losses during encapsulation (ca. $15 \mu \mathrm{M}$ "bulk" or $7.6 \mathrm{mM}$ intravesicular concentration, originating from $10 \mathrm{mM}$ NADH used for encapsulation, diluted $~ 500$ times). Information about the NADH concentration change enables the comparison between the present system and NADH:ubiquinone oxidoreductase (Complex I), which can be regarded as a biological counterpart of TCNQ. If we take as an example the experiment with $200 \mu \mathrm{M}$ ferricyanide during the first 60 min (steep decrease), the average rate of NADH oxidation is about $0.13 \mu \mathrm{M} \mathrm{min}{ }^{-1}$. This value is 
comparable to the reported activity of isolated enzyme $\left(\sim 0.2 \mu \mathrm{M} \mathrm{min}{ }^{-1}{ }^{41}\right)$ but normalizing to the molecular weight of the respective catalysts would result in a better figure of merit.

In the next step we developed a homogeneous phase model to describe the properties of the system. TCNQ is known to undergo two one-electron reductions: to a radical anion $\left(\mathrm{TCNQ}^{-}{ }^{-}\right.$) at $0.21 \mathrm{~V}$ vs. SHE, and subsequently to a dianion $\left(\mathrm{TCNQ}^{2-}\right)$ at $-0.45 \mathrm{~V}$ vs. $\mathrm{SHE}^{42 \text {, }}$ 43. Since the potential of the second redox conversion is more negative than the NADH potential $(-0.32 \mathrm{~V} v s$. SHE), we concluded that the mediator is monoreduced, according to the stoichiometry in Eq. 1. The mechanism of 1-benzyl-1,4-dihydronicotinamide (NADH model compound) oxidation with tetracyanoethylene also involved radical anion ${ }^{44}$. In addition, the electrochemical behavior of immobilized TCNQ during the oxidation of $\mathrm{NADH}^{30}$ or enzymebound $\mathrm{FAD}^{45}$ suggests as well that monoanion species are involved.

$$
N A D H+2 T C N Q^{0} \stackrel{k_{1}}{\rightarrow} N A D^{+}+2 T C N Q^{--}+H^{+}
$$

The mechanism of hydride transfer during NADH oxidation is still not completely elucidated and there is a dichotomy between concerted and stepwise pathways, as shown with $\mathrm{NADH}$ analogues ${ }^{46}$. After the confirmation of the NADH radical cation $\left(\mathrm{NADH} \cdot{ }^{+}\right)^{47}$ it is widely accepted that the chemical oxidation involves a multistep mechanism with consecutive $e^{-} / \mathrm{H}^{+} / e^{-}$steps (see Supplementary Note 1 ). The absence of isotope and $\mathrm{pH}$ effects during the oxidation of NADH by ferrocene ruled out hydride transfer and rate-limiting deprotonation and justified the first electron transfer as a rate determining step. ${ }^{48}$. In the present case we assumed a similar situation and modeled the oxidation of NADH by $\mathrm{TCNQ}^{0}$ through an irreversible bimolecular one-electron transfer with $k_{l, a p p}$ (see Supplementary Note 1).

While pure TCNQ has been rarely used for NADH oxidation ${ }^{30,49}$ (mostly bound in charge transfer complexes ${ }^{45}$ ), it has an established record in combination with ferri/ferrocyanide in $\mathrm{BLM}^{27}$ and ITIES ${ }^{43,50}$ electrochemical setups, according to Eq. 2:

$$
\mathrm{TCN}^{--}+\left[\mathrm{Fe}(\mathrm{CN})_{6}\right]^{3-} \stackrel{K_{e q}}{\longleftrightarrow} \mathrm{TCN}^{0}+\left[\mathrm{Fe}(\mathrm{CN})_{6}\right]^{4-}
$$

where $K_{e q}=k_{2}{ }^{+} / k_{2}{ }^{-}$. Unlike the previous reaction, which has been taken as irreversible due to the large driving force $(\sim 0.53 \mathrm{~V})$ and the known difficulties in chemical reduction of $\mathrm{NAD}^{+}$to $\mathrm{NADH}^{4}$, we considered the regeneration of $\mathrm{TCNQ}^{0}$ as reversible. The redox potential of ferricyanide (around $0.41 \mathrm{~V}$ vs. $\mathrm{SHE}^{51}$ ) lies closer to that of TCNQ, and despite that the reaction form in Eq. 2 is thermodynamically favorable, potential-determining ions can 
influence the equilibrium through a Galvani potential and thus drive uphill electron transfer ${ }^{50}$. This has been confirmed in ITIES (interface between two immiscible electrolyte solutions) systems, which are useful models for the half-reactions in the present case ${ }^{43,52}$. With respect to the partitioning of ions in the bilayer, the ability of TCNQ to transport protons and other cations has been demonstrated through electrochemical experiments in a BLM setup ${ }^{53,54}$, which postulates the generation of additional interfacial potential difference as a plausible phenomenon.

Apart from ferricyanide oxidation, we observed a decrease of the NADH concentration in the absence of added electron acceptor (Fig. 4). As discussed in the previous section, spontaneous NADH degradation took place ${ }^{37}$ but the observed rates were lower (Fig. 2) and therefore the possibility of TCNQ oxidation by dissolved oxygen cannot be ruled out. A computational DFT study has recently revealed that metal salts such as FeTCNQ can efficiently catalyze four-electron oxygen reduction ${ }^{55}$, while in the past it has been shown that electrogenerated TCNQ dianions interact with oxygen to form $\alpha, \alpha$-dicyano- $p$-toluoylcyanide $^{56}$. In addition, TCNQ radical anions (TCNQ ${ }^{-}$), which take part in Eqs. 1 and 2 also exhibit charge transfer interactions with oxygen ${ }^{57}$. Based on this notion we hypothesized the irreversible reduction of oxygen to water as a competing reaction to the ferricyanide reduction (Eq. 3).

$$
2 \mathrm{TCNQ}^{--}+\frac{1}{2} \mathrm{O}_{2}+2 \mathrm{H}^{+} \stackrel{k_{3}}{\rightarrow} 2 \mathrm{TCNQ}^{0}+\mathrm{H}_{2} \mathrm{O}
$$

The redox potentials for oxygen reduction at $\mathrm{pH} 7$ are $0.82 \mathrm{~V}$ and $0.28 \mathrm{~V} v s$. SHE to water and peroxide, respectively ${ }^{21}$, which renders both pathways thermodynamically feasible but the former one more likely. Since oxygen was in excess with respect to TCNQ and the experimental system was buffered we disregarded protons and expressed the reaction through a pseudo second-order kinetics with $k_{3, \text { app }}$ (see Supplementary Note 2).

After definition of the equation system, the experimental data for NADH oxidation were fitted to extract a parameter set (Table 1, initial conditions summarized in Table S2). While the concentrations of $\mathrm{NADH}$, oxygen and ferricyanide were readily available, the homogeneous TCNQ concentration was calculated to $7.4 \mu \mathrm{M}$ ( $\sim 5700$ molecules per vesicle) based on TRPS results $\left(6.99 \times 10^{16} \mathrm{~nm}^{2}\right.$ total outer surface area of vesicles in $1 \mathrm{ml}$, multiplied by 2 to account for two leaflets, $0.63 \mathrm{~nm}^{2}$ area of $\mathrm{POPC}^{58}$, TCNQ:POPC $=1: 50$, assuming uniform distribution of TCNQ). The parameter estimation was performed by minimizing the residual sum of squares (RSS) between simulation and experimental datasets using the 
Table 1: Estimated rate and equilibrium constants.

\begin{tabular}{|c|c|c|}
\hline Parameter & Fitted value & Confidence interval \\
\hline$k_{1, a p p}$ & $2.58 \times 10^{2} \mathrm{M}^{-1} \mathrm{~s}^{-1}$ & $\pm 1.6 \times 10^{2}$ \\
\hline$K_{e q}$ & $5.7 \times 10^{-3}$ & $\pm 2.2 \times 10^{-3}$ \\
\hline$k_{3, \text { app }}$ & $4.2 \mathrm{M}^{-1} \mathrm{~s}^{-1}$ & \pm 1.2 \\
\hline
\end{tabular}

The fitted curves described well the experimental data (Fig. 4) but it is not straightforward to compare the obtained constants with literature values because the latter have been expressed in different ways. For instance, the rate constant for NADH oxidation by TCNQ in an amperometric sensor has been determined to $4.8 \times 10^{6} \mathrm{M}^{-1} \mathrm{~s}^{-1}$ based on peak current analysis $^{30}$. It should be noted that this work used unconventional reaction scheme expression through an electrically unbalanced reaction between NADH and TCNQ, while the typical scheme for mediated enzymatic electrodes is neutral and accounts for the respective electron stoichiometry as in Eq. $1^{49}$. The rate constants greatly varied in other homogeneous systems. For instance, the oxidation of NADH by ferrocene was previously determined to be 50 times slower than in the present case $\left(5.4 \mathrm{M}^{-1} \mathrm{~s}^{-1}\right)^{48}$ but when radiolytically generated dibromide radical anion was used as oxidant the rate constant was 7 orders of magnitude higher (1.1$\left.1.5 \times 10^{9} \mathrm{M}^{-1} \mathrm{~s}^{-1}\right)^{63}$.

On the contrary, the obtained $K_{e q}$, characterizing Eq. 2, was in the range of values, resulting from the expected concentrations of species, having in mind the excess of ferricyanide. In addition, we transformed $K_{e q}$ into electron transfer rate constant in order to compare it with existing values in ITIES setups. The Marcus cross-relation ${ }^{64}$ allows for calculation of homogeneous electron transfer rate constant, according to: $k_{12}=$ $\left(k_{11} k_{22} K_{12} f_{12}\right)^{0.5} W_{12}$, where $k_{12}, K_{12}$ are analogous to $k_{2}{ }^{+}, K_{e q}$ in the present case. The contributions of various work terms for reactants and products expressed in the parameters $f_{12}$ (known as frequency factor) and $W_{12}$ are typically close to 1 and thus $f_{12}, W_{12}=1$ is a customary assumption $^{65}$. The self-exchange rate constants $\left(k_{11}, k_{22}\right)$ for TCNQ/TCNQ.- and 
ferri/ferrocyanide have been cited to $10^{9} \mathrm{M}^{-1} \mathrm{~s}^{-1}$ and $5.8 \times 10^{4} \mathrm{M}^{-1} \mathrm{~s}^{-1}$, respectively ${ }^{52}$. We divided the obtained homogeneous $k_{12}$ by the surface-to-volume ratio of the bilayer (relationship derived from mass balance) to convert it to a heterogeneous rate constant and calculated a value of $0.27 \mathrm{~cm} \mathrm{M}^{-1} \mathrm{~s}^{-1}$. In addition, since it is known that the self-exchange reaction of ferri/ferrocyanide is influenced by cations, we used a cation-independent $k_{22}$ value of lower magnitude $\left(2.4 \times 10^{2} \mathrm{M}^{-1} \mathrm{~s}^{-1}\right)^{66}$, and obtained $c a$. 15 times lower value $\left(0.018 \mathrm{~cm} \mathrm{M}^{-1}\right.$ $\mathrm{s}^{-1}$ ) for the heterogeneous bimolecular rate constant.

The latter has been previously determined for TCNQ/ferricyanide by impedance analysis in ITIES setup to $0.129 \mathrm{~cm} \mathrm{M}^{-1} \mathrm{~s}^{-1}$ based on apparent standard rate constant of $5.4 \times 10^{-3} \mathrm{~cm}$ $\mathrm{s}^{-167}$. The same authors reported decreased apparent rate constant of $1.8 \times 10^{-3} \mathrm{~cm} \mathrm{~s}^{-1}$ in the presence of adsorbed phospholipid monolayer at the interface and discussed it in the context of the Markus theory ${ }^{43}$. It should be noted, that the heterogeneous bimolecular constant $(0.129$ $\mathrm{cm} \mathrm{M}^{-1} \mathrm{~s}^{-1}$ ) has been defined at zero driving force (referred to as standard) in the context of the Butler-Volmer theory ${ }^{67}$. Thus, in the expression for $K_{\text {eq }}$, the bimolecular constants cancel out and $K_{e q}$ is dependent only on the driving force, expressed as potential, which we determined to around $-130 \mathrm{mV}$ in the present case. Assuming transfer coefficient of 0.5 , the driving force of $-130 \mathrm{mV}$ was used to transform the literature standard rate constant to a forward rate constant ${ }^{52}\left(\sim 0.01 \mathrm{~cm} \mathrm{M}^{-1} \mathrm{~s}^{-1}\right)$ to be able to compare it with the values calculated through the Marcus cross-relation $\left(0.27\right.$ or $\left.0.018 \mathrm{~cm} \mathrm{M}^{-1} \mathrm{~s}^{-1}\right)$, which included the driving force by definition and neglected work-related terms. As can be seen, the orders of magnitude roughly correspond to each other but we will refrain from further conclusions due to the different experimental setups and made assumptions. With respect to the equilibrium, Barker et al. have studied its manipulation through partitioning ions and reported a useful Tafel relationship for $k_{2}{ }^{+}$and $k_{2}{ }^{-}$. However, direct reading of the driving force in the present case is obstructed, since their scale was defined with respect to the partitioning perchlorate ion ${ }^{52}$.

Despite the simplicity of the developed model, as it did not include thermodynamic parameters or mass transport, it exhibited a useful predictive power also in regard to certain experimental effects. It was not possible to find a feasible parameter set under the assumption that the total amount of TCNQ was initially uncharged. Therefore, we supposed that during the vesicle preparation a certain amount was reduced to TCNQ ${ }^{-}$. By estimating the initial conditions, we found that approximately $85 \%$ of the total TCNQ pool was in the form of a radical anion (Table S2). Indeed, the spontaneous reduction of TCNQ in Langmuir-Blodgett monolayers has been reported and discussed in terms of stabilizing effects of the lipid cationic head group and the interfacial hydroxyl group concentration ${ }^{68}$. According to our simulation 
results, TCNQ ${ }^{-}$was reoxidized when ferricyanide was added to the vesicle suspension, assuming a range of values for $k_{2}^{-}$(e.g. Fig. S7). This effect increased the pool of active mediator and enhanced the NADH oxidation, according to Eq. 1.

\section{Redox states of TCNQ}

To determine the redox state of TCNQ in the present setup, we monitored the absorbance of rehydrated phospholipid suspensions before extrusion in presence of the two oxidants (ferricyanide and oxygen). For these experiments we used four times higher TCNQ concentration (50:4 molar ratio between lipids and mediator) in order to magnify the respective signals. Neutral TCNQ in methanol exhibits a maximum at $395 \mathrm{~nm}$, which corresponds to the transition from ground to first excited state $\left(\mathrm{S}_{0} \rightarrow \mathrm{S}_{1}\right)$ (Fig. S8). In the case of the TCNQ anion there are two main bands - one at $420 \mathrm{~nm}\left(\mathrm{D}_{0} \rightarrow \mathrm{D}_{2}\right)$ and a broader one with two maxima at 740 and $840 \mathrm{~nm}\left(\mathrm{D}_{0} \rightarrow \mathrm{D}_{1}\right)$, corresponding to doublet transitions due to the unpaired single electron ${ }^{69}$. The spectrum of the suspension after rehydration revealed the characteristic TCNQ radical anion features - a pronounced band above $600 \mathrm{~nm}$ and peak at $425 \mathrm{~nm}$ with a lower magnitude (Fig. 5a). This proved the spontaneous reduction during preparation, anticipated by the estimation of initial conditions and observed previously ${ }^{68}$. After addition of $400 \mu \mathrm{M}$ ferricyanide to the sample the peaks corresponding to $S_{0} \rightarrow S_{1}$ or $\mathrm{D}_{0} \rightarrow \mathrm{D}_{2}$ transitions could not be analysed because they were masked by the oxidant but the $>600 \mathrm{~nm}$ band was immediately reduced $(<1 \mathrm{sec})$, which manifested oxidation of TCNQ ${ }^{-}$, as predicted by the model. In a parallel experiment the initial TCNQ/lipid suspension was aerated with oxygen for $5 \mathrm{~h}$. The decrease of the $\mathrm{D}_{0} \rightarrow \mathrm{D}_{1}$ band (Fig. $5 \mathrm{~b}$ ) indicated TCNQ ${ }^{-}$ oxidation, consistent with the outcome of the vesicle experiments (Fig. 4). 

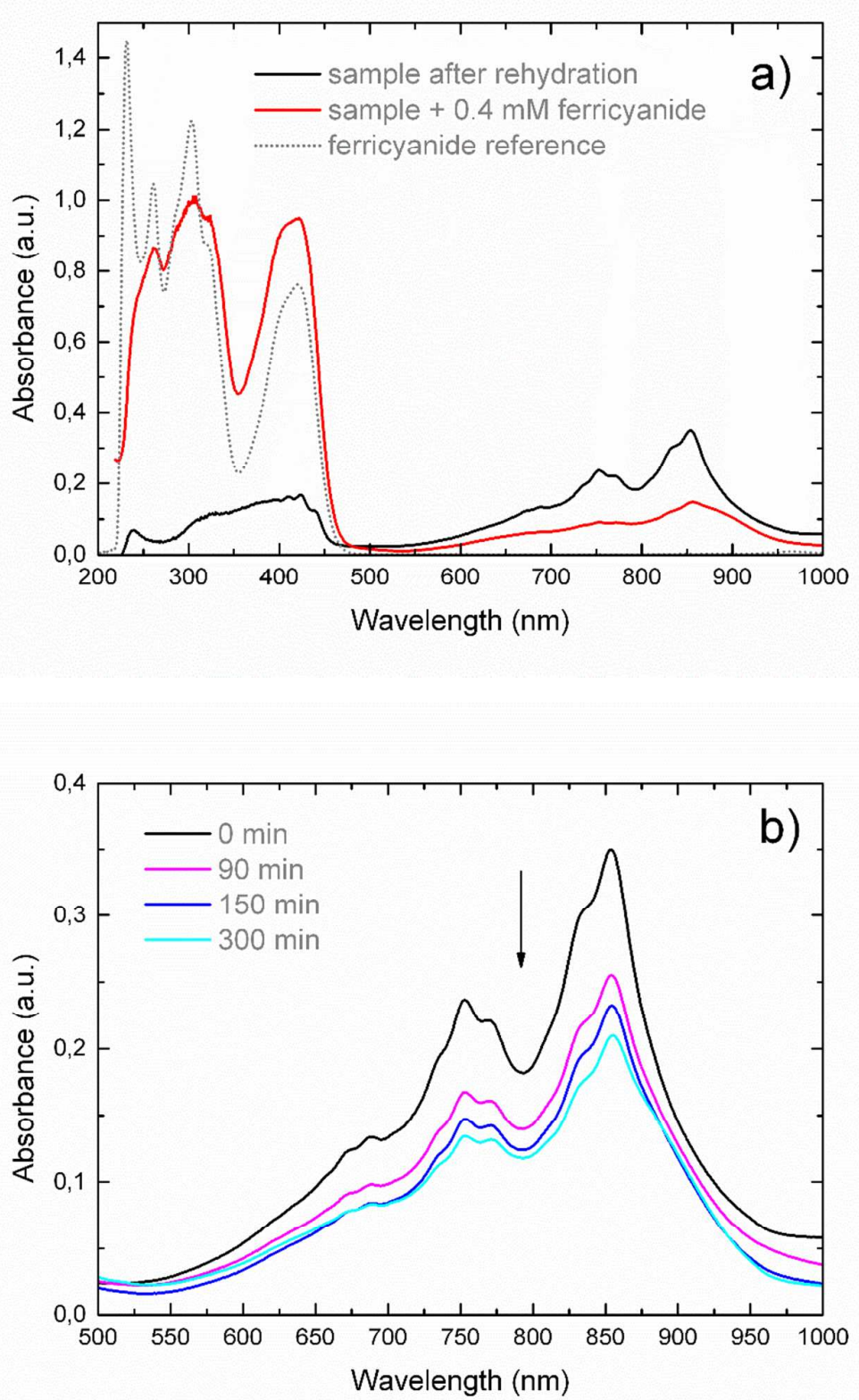

Figure 5: Absorbance spectra of hydrated POPC/TCNQ suspensions a) in presence of $400 \mu \mathrm{M}$ potassium ferricyanide and b) during aeration with oxygen. $10 \mathrm{mM}$ POPC, $0.8 \mathrm{mM}$ TCNQ in $100 \mathrm{mM}$ MOPS, $250 \mathrm{mM}$ $\mathrm{KCl}$, Tris, $\mathrm{pH}$ 7.2.

\section{Conclusions and Outlook}

We have demonstrated a feasible strategy for the transmembrane oxidation of encapsulated NADH by externally added electron acceptor without the involvement of proteins, and described the system behaviour through a spatially lumped homogeneous model. The primary motivation behind this approach was the utilization as $\mathrm{NAD}^{+}$regeneration strategy for enzymatic oxidations but the system also holds conceivable potential for use in living cells 
with respect to redox tuning of the NAD pool ${ }^{70}$. The theoretical description resulted in consistency with literature information and predicted a spontaneous TCNQ reduction effect. In this work we substantiated the chemical aspect of "synthetic" in synthetic biology, opposed to the common interpretation in terms of man-made design in the top-down approach. A plain molecule like TCNQ allowed us to mimic certain electron transfer chain functionalities through a special form of interfacial redox catalysis, found in living cells. In this respect, replacement of natural electron shuttles with synthetic counterparts provides the basis for novel architectures and opens new process design possibilities in the stepwise assembly of minimal cells for biotechnological applications.

The results display the potential of the presented concept but logically raise additional questions. From an experimental point of view the next logical step (already undertaken) is encapsulation of an NAD-dependent dehydrogenase to demonstrate the utility of our approach for continuous cofactor regeneration. Experiments for more precise elucidation of the mechanism would be also beneficial. Regarding the better theoretical description of the system that we are striving for, it will be useful to expand the rate expressions with electron transfer parameters such as potential, according to Butler-Volmer kinetics or Markus theory. In this way we would be able to account for interfacial potential effects due to e.g. transport of protons. Another important aspect that has to be considered is the influence of mass transport in this obviously heterogeneous system, whereby the interface plays a leading role. It remains to be seen how much the higher physicochemical precision would improve the predictability of the model but it would provide a solid engineering framework for membrane electron transfer phenomena in (synthetic) biological systems.

\section{Acknowledgements}

This work is part of the MaxSynBio consortium, which is jointly funded by the Federal Ministry of Education and Research of Germany (BMBF) and the Max Planck Society (MPG).

\section{Supporting Information}

NADH and carboxyfluorescein fluorescence, micrograph of phospholipid suspension, size and concentration of vesicle suspensions, initial conditions for simulation, TCNQ and LiTCNQ absorbance, mechanism of NADH oxidation, rate expressions used in model.

\section{References}


(1) Chiarugi, A.; Dolle, C.; Felici, R.; Ziegler, M. The NAD Metabolome - a Key Determinant of Cancer Cell Biology. Nat. Rev. Cancer 2012, 12, 741-752.

(2) Lin, S. J.; Guarente, L. Nicotinamide Adenine Dinucleotide, a Metabolic Regulator of Transcription, Longevity and Disease. Curr. Opin. Cell Biol. 2003, 15, 241-246.

(3) Houtkooper, R. H.; Canto, C.; Wanders, R. J.; Auwerx, J. The Secret Life of NAD+: an Old Metabolite Controlling New Metabolic Signaling Pathways. Endocr. Rev. 2010, 31, 194-223.

(4) Wu, H.; Tian, C.; Song, X.; Liu, C.; Yang, D.; Jiang, Z. Methods for the Regeneration of Nicotinamide Coenzymes. Green Chem. 2013, 15, 1773-1789.

(5) van der Donk, W. A.; Zhao, H. Recent Developments in Pyridine Nucleotide Regeneration. Curr. Opin. Biotechnol. 2003, 14, 421-426.

(6) Schwille, P. Bottom-Up Synthetic Biology: Engineering in a Tinkerer's World. Science 2011, 333, 1252 1254.

(7) Kuchler, A.; Yoshimoto, M.; Luginbuhl, S.; Mavelli, F.; Walde, P. Enzymatic Reactions in Confined Environments. Nat. Nanotechnol 2016, 11, 409-420.

(8) Elani, Y.; Law, R. V.; Ces, O. Vesicle-based Artificial Cells as Chemical Microreactors with Spatially Segregated Reaction Pathways. Nat. Commun. 2014, 5, 5305.

(9) Noireaux, V.; Libchaber, A. A vesicle Bioreactor as a Step toward an Artificial Cell Assembly. PNAS 2004, $101,17669-17674$.

(10) Kurihara, K.; Tamura, M.; Shohda, K.; Toyota, T.; Suzuki, K.; Sugawara, T. Self-reproduction of Supramolecular Giant Vesicles Combined with the Amplification of Encapsulated DNA. Nat. Chem. 2011, 3, $775-781$.

(11) Walde, P.; Ichikawa, S. Enzymes inside Lipid Vesicles: Preparation, Reactivity and Applications. Biomol. Eng. 2001, 18, 143-177.

(12) Yoshimoto, M.; Yamashita, T.; Yamashiro, T. Stability and Reactivity of Liposome-Encapsulated Formate Dehydrogenase and Cofactor System in Carbon Dioxide Gas-Liquid Flow. Biotechnol. Prog. 2010, 26, 10471053.

(13) Yoshimoto, M.; Sato, M.; Yoshimoto, N.; Nakao, K. Liposomal Encapsulation of Yeast Alcohol Dehydrogenase with Cofactor for Stabilization of the Enzyme Structure and Activity. Biotechnol. Prog. 2008, $24,576-582$.

(14) Zhang, X. Y.; Lomora, M.; Einfalt, T.; Meier, W.; Klein, N.; Schneider, D.; Palivan, C. G. Active Surfaces Engineered by Immobilizing Protein-polymer Nanoreactors for Selectively Detecting Sugar Alcohols. Biomaterials 2016, 89, 79-88.

(15) Matsumoto, R.; Kakuta, M.; Sugiyama, T.; Goto, Y.; Sakai, H.; Tokita, Y.; Hatazawa, T.; Tsujimura, S.; Shirai, O.; Kano, K. A liposome-based Energy Conversion System for Accelerating the Multi-enzyme Reactions. Phys. Chem. Chem. Phys. 2010, 12, 13904-13906.

(16) Fujita, S.; Matsumoto, R.; Ogawa, K.; Sakai, H.; Maesaka, A.; Tokita, Y.; Tsujimura, S.; Shirai, O.; Kano, K. Bioelectrocatalytic oxidation of glucose with antibiotic channel-containing liposomes. Phys. Chem. Chem. Phys. 2013, 15, 2650-2653.

(17) Robinson, J. N.; Colehamilton, D. J. Electron-Transfer across Vesicle Bilayers. Chem. Soc. Rev. 1991, 20, 49-94. 
(18) Hinkle, P. A Model System for Mitochondrial Ion Transport and Respriratory Control. Biochem. Biophys. Res. Commun. 1970, 41, 1375-1381.

(19) Takeoka, S.; Ohgushi, T.; Nishide, H.; Tsuchida, E. Reduction of Methemoglobin via Electron Transfer across the Bilayer Membrane of hb Vesicles. Bull. Chem. Soc. Jpn. 1997, 70, 1171-1178.

(20) Melby, L. R.; Mahler, W.; Mochel, W. E.; Harder, R. J.; Hertler, W. R.; Benson, R. E. Substituted Quinodimethans .2. Anion-radical Derivatives and Complexes of 7,7,8,8-Tetracyanoquinodimethan. JACS 1962, 84, 3374-3387.

(21) Wood, P. M. The Potential Diagram for Oxygen at pH 7. Biochem. J. 1988, 253, 287-289.

(22) Schafer, F. Q.; Buettner, G. R. Redox Environment of the Cell as Viewed through the Redox State of the Glutathione Disulfide/Glutathione Couple. Free Radical Biol. Med. 2001, 30, 1191-1212.

(23) Daskalakis, N. N.; Muller, A.; Evans, S. D.; Jeuken, L. J. C. Driving Bioenergetic Processes with Electrodes. Soft Matter 2011, 7, 49-52.

(24) Cheng, Y. F.; Cunnane, V. J.; Kontturi, A. K.; Kontturi, K.; Schiffrin, D. J. Potential Dependence of Transmembrane Electron Transfer across Phospholipid Bilayers Mediated by Ubiquinone 10. J. Phys. Chem. 1996, 100, 15470-15477.

(25) Campos, R.; Kataky, R. Electron Transport in Supported and Tethered Lipid Bilayers Modified with Bioelectroactive Molecules. J. Phys. Chem.. B 2012, 116, 3909-17.

(26) Ma, W.; Li, D.-W.; Sutherland, T. C.; Li, Y.; Long, Y.-T.; Chen, H.-Y. Reversible Redox of NADH and $\mathrm{NAD}(+)$ at a Hybrid Lipid Bilayer Membrane Using Ubiquinone. JACS 2011, 133, 12366-12369.

(27) Tien, H. T. Cyclic Voltammetry of Electron-conducting Bilayer Lipid-membranes. Bioelectrochem. Bioenerg. 1984, 13, 299-316.

(28) Shiba, H.; Maeda, K.; Ichieda, N.; Kasuno, M.; Yoshida, Y.; Shirai, O.; Kihara, S. Voltammetric Study on the Electron Transport through a Bilayer Lipid Membrane Containing Neutral or Ionic Redox Molecules. $J$. Electroanal. Chem. 2003, 556, 1-11.

(29) Asaka, K.; Ottova, A.; Tien, H. T. Mediated Electron Transfer across Supported Bilayer Lipid Membrane (s-BLM). Thin Solid Films 1999, 354, 201-207.

(30) Murthy, A. S. N.; Gupta, A. R. L. NADH Sensor with Electrochemically Modified TCNQ Electrode. Anal. Chim. Acta 1994, 289, 43-46.

(31) Lemmetyinen, H.; Yliperttula, M.; Mikkola, J.; Kinnunen, P. Quenching of Fluorescence of Pyrenesubstituted Lecithin by Tetracyanoquinodimethane in Liposomes. Biophys. J. 1989, 55, 885-895.

(32) Gorton, L.; Dominguez, E. Electrocatalytic Oxidation of NAD(P)H at Mediator-modified Electrodes. $J$. Biotechnol. 2002, 82, 371-392.

(33) Sevcik, P. D. B. The Rate Constant for Aerial Oxidation of NADH by Methylene Blue. Int. J. Chem. Kinet. 1995, 27, 925-928.

(34) Kruk, J.; Strzalka, K.; Leblanc, R. M. Fluorescence Properties of Plastoquinol, Ubiquinol and Alphatocopherol Quinol in Solution and Liposome Membranes. J. Photochem. Photobiol., B 1993, 19, 33-38.

(35) Tamaya, H.; Nakano, H.; Iimori, T. 7,7,8,8-Tetracyanoquinodimethane (TCNQ) Emits Visible Photoluminescence in Solution. J. Lumin. 2017, 192, 203-207.

(36) Hernandez, V. A.; Eriksson, E. K.; Edwards, K. Ubiquinone-10 Alters Mechanical Properties and Increases Stability of Phospholipid Membranes. Biochim. Biophys. Acta, Biomembr. 2015, 1848, 2233-2243. 
(37) Rover Junior, L.; Fernandes, J. C.; de Oliveira Neto, G.; Kubota, L. T.; Katekawa, E.; Serrano, S. H. Study of NADH Stability Using Ultraviolet-visible Spectrophotometric Analysis and Factorial Design. Anal. Biochem. 1998, 260, 50-5.

(38) Chen, X. W.; Qi, F.; Dash, R. K.; Beard, D. A. Kinetics and Regulation of Mammalian NADH-Ubiquinone Oxidoreductase (Complex I). Biophys. J. 2010, 99, 1426-1436.

(39) Weinstein, J. N.; Blumenthal, R.; Klausner, R. D. Carboxyfluorescein Leakage Assay for Lipoproteinliposome Interaction. Methods Enzymol. 1986, 128, 657-668.

(40) De Maria, P.; Filippone, P.; Fontana, A.; Gasbarri, C.; Siani, G.; Velluto, D. Cardanol as a Replacement for Cholesterol into the Lipid Bilayer of POPC Liposomes. Colloids Surf., B 2005, 40, 11-18.

(41) Kussmaul, L.; Hirst, J. The Mechanism of Superoxide Production by NADH:ubiquinone Oxidoreductase (complex I) from Bovine Heart Mitochondria. PNAS 2006, 103, 7607-7612.

(42) Le, T. H.; Nafady, A.; Qu, X. H.; Bond, A. M.; Martin, L. L. Redox and Acid-Base Chemistry of 7,7,8,8Tetracyanoquinodimethane, 7,7,8,8-Tetracyanoquinodimethane Radical Anion, 7,7,8,8Tetracyanoquinodimethane Dianion, and Dihydro-7,7,8,8-Tetracyanoquinodimethane in Acetonitrile. Anal. Chem. 2012, 84, 2343-2350.

(43) Cheng, Y. F.; Schiffrin, D. J. Redox Electrocatalysis by Tetracyanoquinodimethane in Phospholipid Monolayers Absorbed at a Liquid-liquid Interface. J. Chem. Soc., Faraday Trans 1994, 90, 2517-2523.

(44) Fukuzumi, S.; Kondo, Y.; Tanaka, T. Effects of Base on Oxidation of an NADH Model-compound by Iron(III) Complexes and Tetracyanoethylene. J. Chem. Soc., Perkin Trans. 2 1984, 673-679.

(45) Ivanov, I.; Vidakovic-Koch, T.; Sundmacher, K. Alternating Electron Transfer Mechanism in the Case of High-performance Tetrathiafulvalene-tetracyanoquinodimethane Enzymatic Electrodes. J. Electroanal. Chem. 2013, 690, 68-73.

(46) Yuasa, J.; Fukuzumi, S. A mechanistic Dichotomy in Concerted versus Stepwise Pathways in Hydride and Hydrogen Transfer Reactions of NADH Analogues. J. Phys. Org. Chem. 2008, 21, 886-896.

(47) Czochralska, B.; Lindqvist, L. Biphotonic One-electron Oxidation of NADH on Laser Excitation at $353 \mathrm{~nm}$. Chem. Phys. Lett. 1983, 101, 297-299.

(48) Carlson, B. W.; Miller, L. L.; Neta, P.; Grodkowski, J. Oxidation of NADH Involving Rate-limiting Oneelectron Transfer. JACS 1984, 106, 7233-7239.

(49) Pandey, P. C. Tetracyanoquinodimethane-mediated Flow-injection Analysis Electrochemical Sensor for NADH Coupled with Dehydrogenase Enzymes. Anal. Biochem. 1994, 221, 392-396.

(50) Solomon, T.; Bard, A. J. Reverse (Uphill) Electron-transfer at the Liquid/Liquid Interface. J. Phys. Chem. 1995, 99, 17487-17489.

(51) Oreilly, J. E. Oxidation-reduction Potential of Ferro-ferricyanide System in Buffer Solutions. Biochim. Biophys. Acta 1973, 292, 509-515.

(52) Barker, A. L.; Unwin, P. R.; Zhang, J. Measurement of the Forward and Back Rate Constants for Electron Transfer at the Interface between Two Immiscible Electrolyte Solutions Using Scanning Electrochemical Microscopy (SECM): Theory and Experiment. Electrochem. Commun. 2001, 3, 372-378.

(53) Hichiri, K.; Shirai, O.; Kitazumi, Y.; Kano, K. Coupling of Proton Transport across Planar Lipid Bilayer and Electron Transport Catalyzed by Membrane-bound Enzyme D-Fructose Dehydrogenase. Electrochemistry 2016, 84, 328-333. 
(54) Sasakura, K.; Shirai, O.; Hichiri, K.; Goda-Tsutsunii, M.; Tsujimura, S.; Kano, K. Ion Transport across Planar Bilayer Lipid Membrane Driven by D-Fructose Dehydrogenase-catalyzed Electron Transport. Chem. Lett. 2011, 40, 486-488.

(55) Wang, N.; Feng, L. Y.; Shang, Y. C.; Zhao, J. X.; Cai, Q. H.; Jin, P. Two-dimensional Irontetracyanoquinodimethane (Fe-TCNQ) Monolayer: an Efficient Electrocatalyst for the Oxygen Reduction Reaction. RSC Adv. 2016, 6, 72952-72958.

(56) Suchanski, M. R.; Vanduyne, R. P. Resonance Raman Spectroelectrochemistry .4. Oxygen Decay Chemistry of Tetracyanoquinodimthane Dianion. JACS 1976, 98, 250-252.

(57) Sakata, T.; Okai, T.; Tsubomura, H. Charge-transfer Interactions between Oxygen and Anion Radicals of Tetracyanoquinodimthane and Tetracyanoethylene. Bull. Chem. Soc. Jpn. 1975, 48, 2207-2208.

(58) Kucerka, N.; Nieh, M. P.; Katsaras, J. Fluid Phase Lipid Areas and Bilayer Thicknesses of Commonly Used Phosphatidylcholines as a Function of Temperature. Biochim. Biophys. Acta, Biomembr. 2011, 1808, 27612771 .

(59) Hoops, S.; Sahle, S.; Gauges, R.; Lee, C.; Pahle, J.; Simus, N.; Singhal, M.; Xu, L.; Mendes, P.; Kummer, U. COPASI- A COmplex PAthway SImulator. Bioinformatics 2006, 22, 3067-3074.

(60) Back, T.; Schwefel, H. P. An Overview of Evolutionary Algorithms for Parameter Optimization. Evol. Comput. 1993, 1, 1-23.

(61) Nelder, J. A.; Mead, R. A Simplex-method for Function Minimization. Comput. J. 1965, 7, 308-313.

(62) Schaber, J. Easy Parameter Identifiability Analysis with COPASI. Biosystems 2012, 110, 183-185.

(63) Zielonka, J.; Marcinek, A.; Adamus, J.; Gebicki, J. Direct Observation of NADH Radical Cation Generated in Reactions with One-electron Oxidants. J. Phys. Chem. A 2003, 107, 9860-9864.

(64) Marcus, R. A.; Sutin, N. Electron Transfers in Chemistry and Biology. Biochim. Biophys. Acta 1985, 811, $265-322$.

(65) Sethi, R.; Sangaranarayanan, M. V. Nonequilibrium Thermodynamics Formalism for Marcus Theory of Heterogeneous and Self-exchange Electron-transfer Rate Constants. J. Phys. Chem. A 2008, 112, 4308-4313.

(66) Zahl, A.; van Eldik, R.; Swaddle, T. W. Cation-dependent Electron Transfer between Ferricyanide and Ferrocyanide Ions in Aqueous Solution. Inorg. Chem. 2002, 41, 757-764.

(67) Cheng, Y. F.; Schiffrin, D. J. AC Impedance Study of Rate Constants for 2 Phase Electron-transfer Reactions. J. Chem. Soc., Faraday Trans. 1993, 89, 199-205.

(68) Ahuja, R. C.; Dringenberg, B. J. Reduction of TCNQ in Mixed Monolayers with Cationic Amphiphiles at the Air/Water Interface. Langmuir 1995, 11, 1515-1523.

(69) Ma, L.; Hu, P.; Jiang, H.; Kloc, C.; Sun, H. D.; Soci, C.; Voityuk, A. A.; Michel-Beyerle, M. E.; Gurzadyan, G. G. Single photon triggered dianion formation in TCNQ and F(4)TCNQ crystals. Sci. Rep. 2016, 6, 28510

(70) Liu, Z.; Romero-Canelon, I.; Qamar, B.; Hearn, J. M.; Habtemariam, A.; Barry, N. P. E.; Pizarro, A. M.; Clarkson, G. J.; Sadler, P. J. The Potent Oxidant Anticancer Activity of Organoiridium Catalysts. Angew. Chem. Int. Ed. 2014, 53, 3941-3946. 
For Table of Contents Use Only
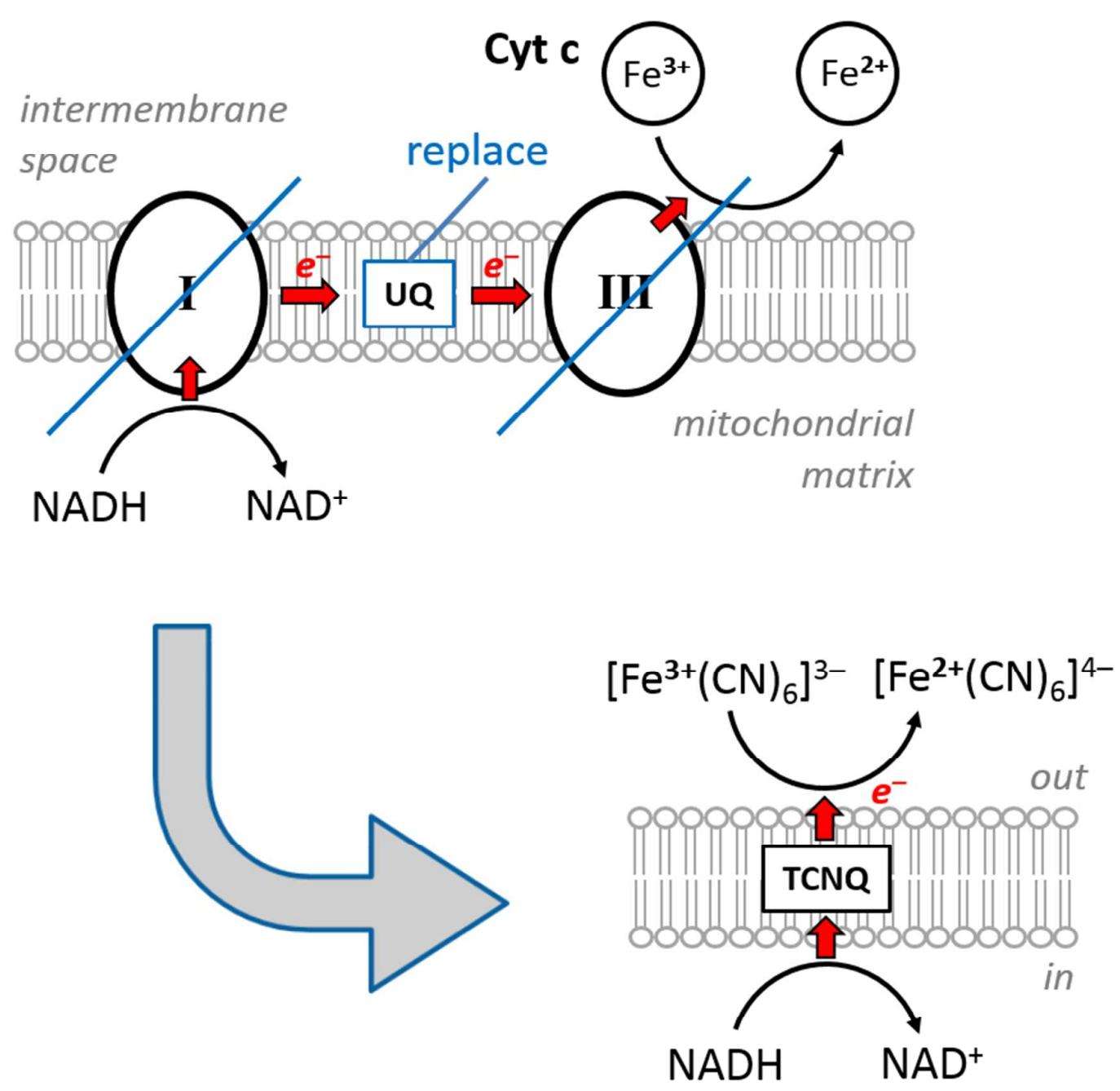

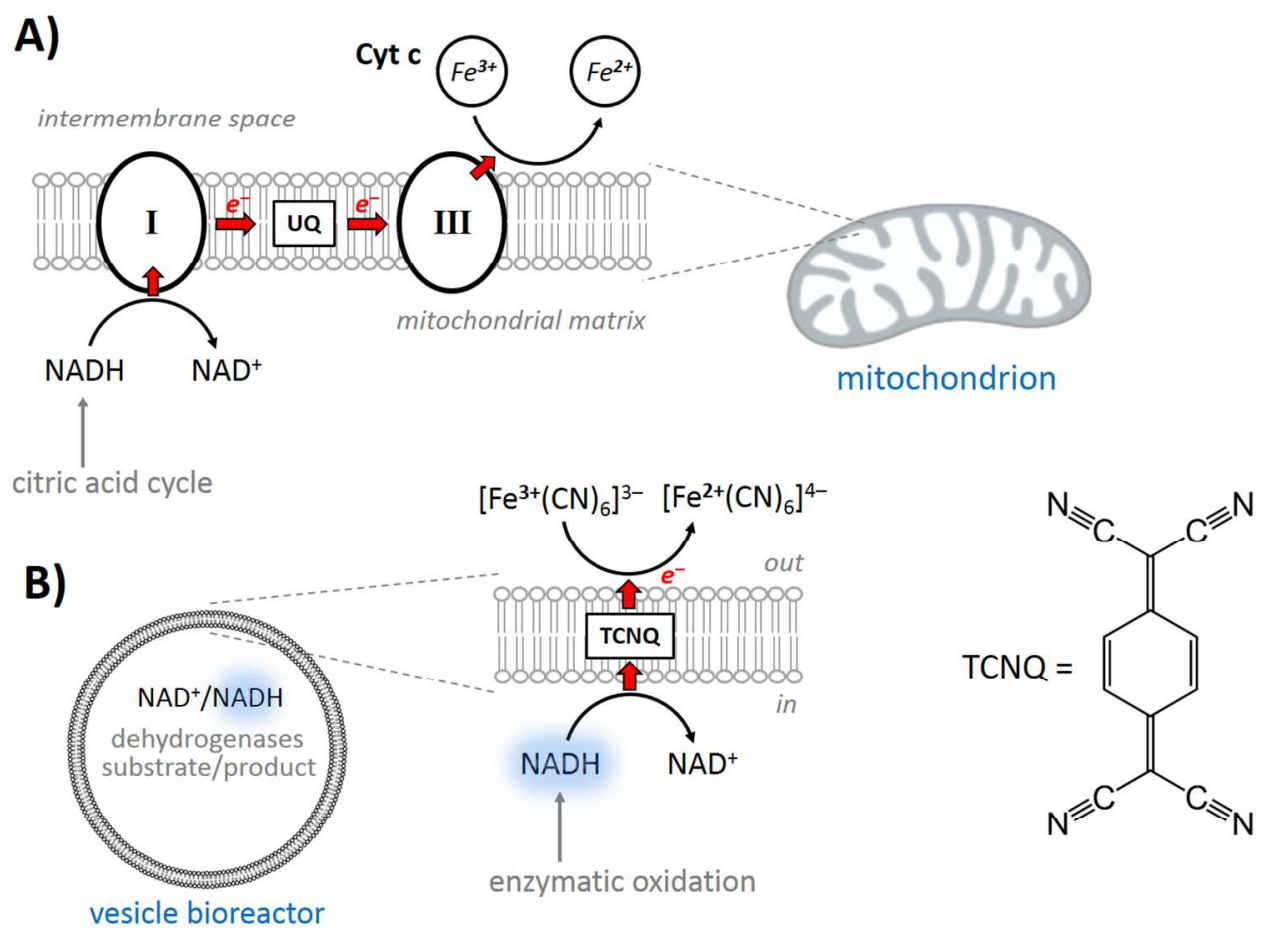

$251 \times 186 \mathrm{~mm}(150 \times 150 \mathrm{DPI})$ 


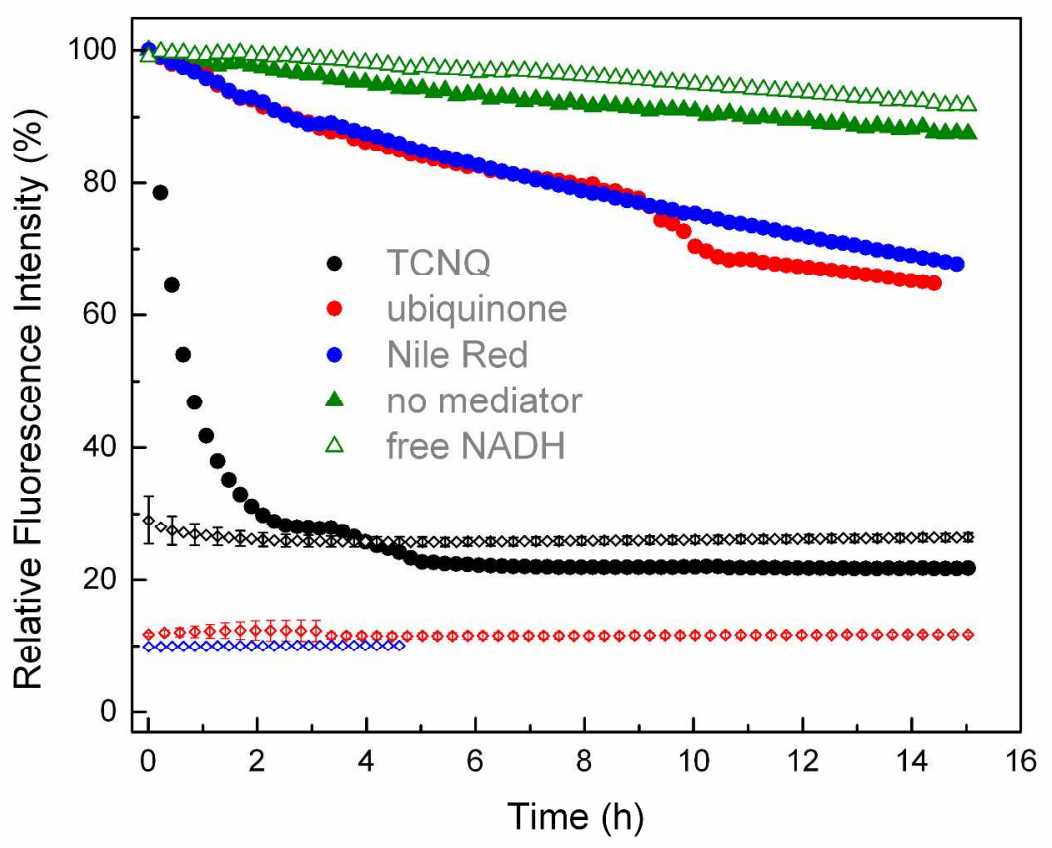

$272 \times 208 \mathrm{~mm}(300 \times 300$ DPI $)$ 


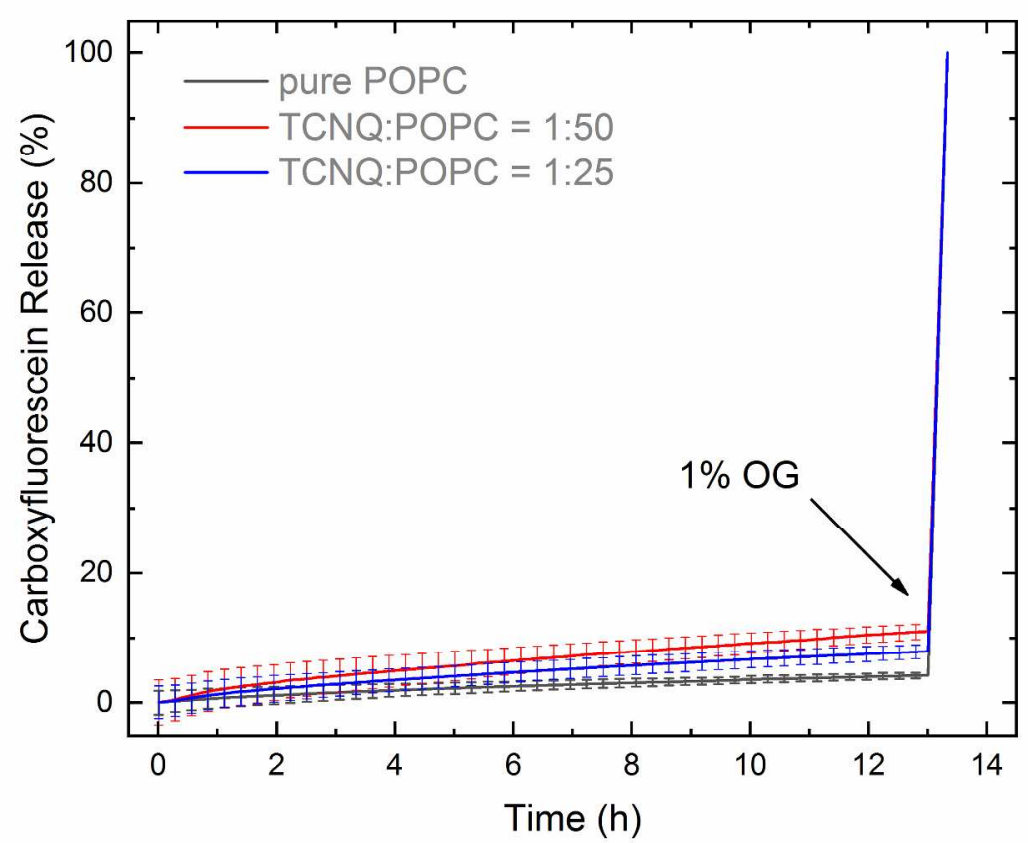

$272 \times 208 \mathrm{~mm}(300 \times 300$ DPI) 


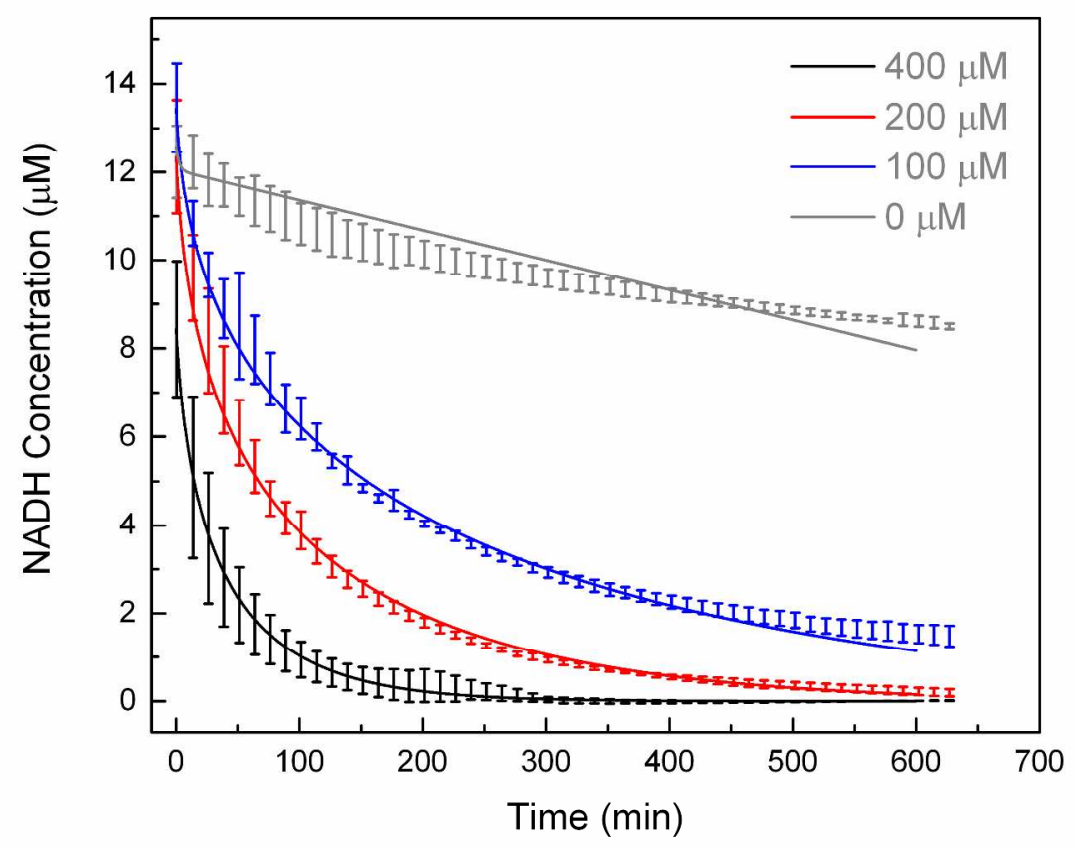

$272 \times 208 \mathrm{~mm}(300 \times 300$ DPI $)$ 


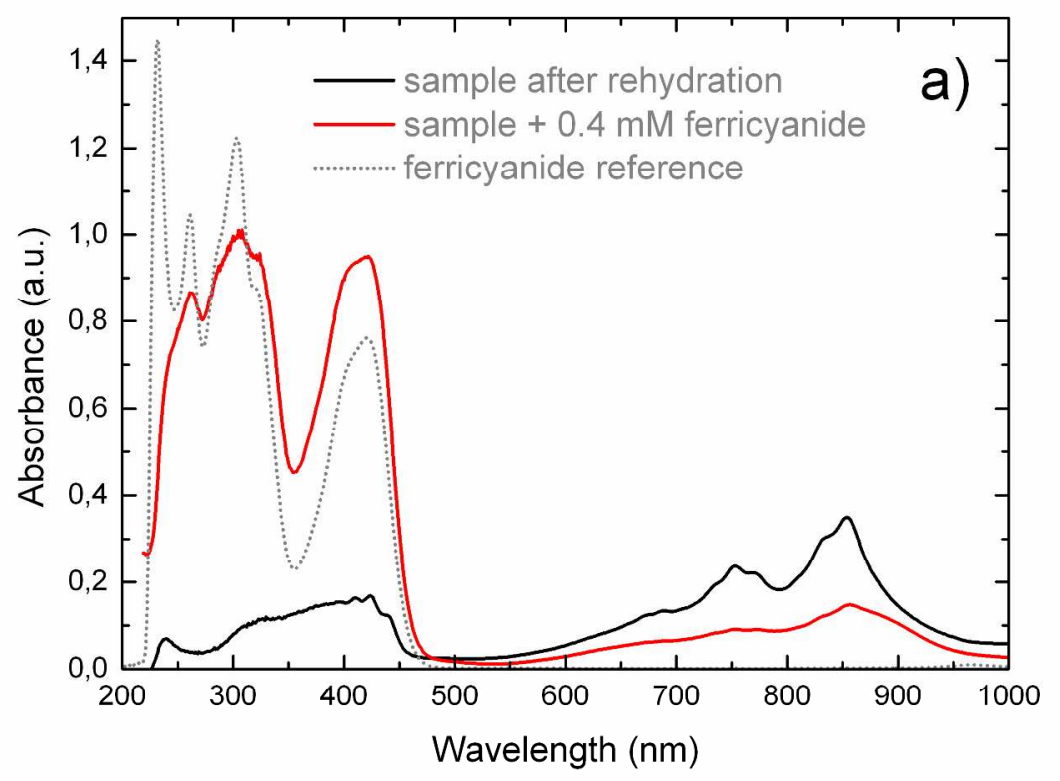

$286 \times 199 m m(300 \times 300$ DPI $)$ 


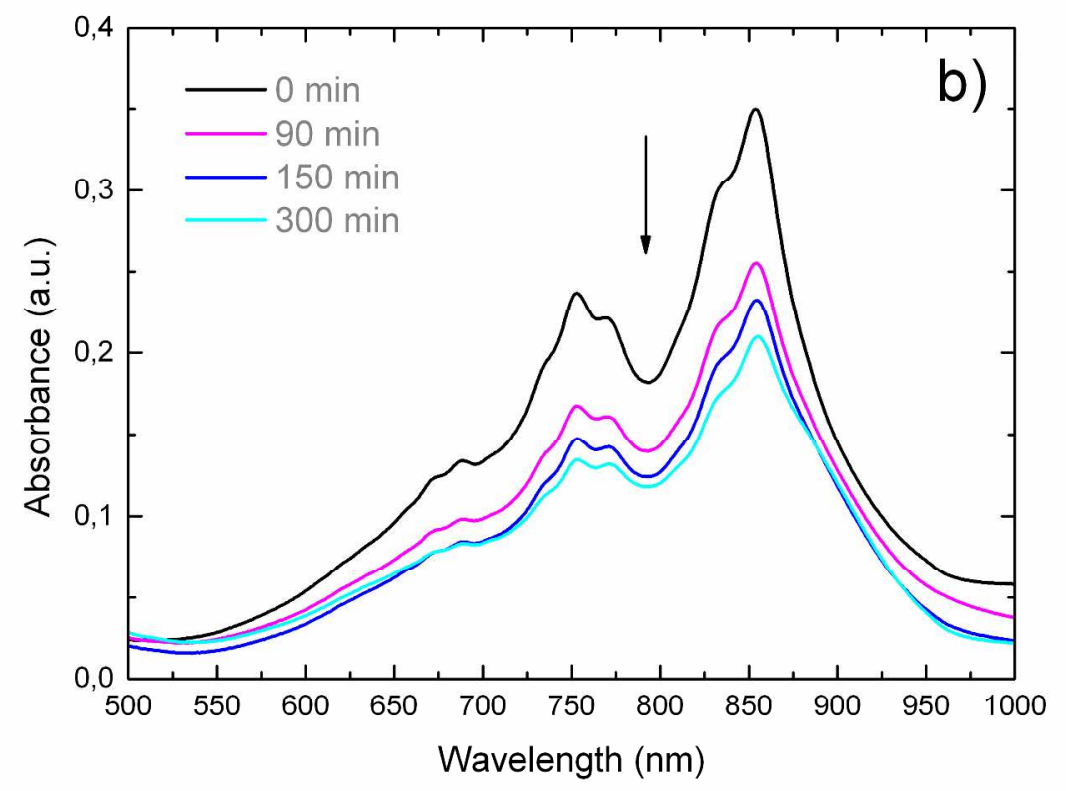

286x199mm (300 x 300 DPI) 